\title{
AVALIAÇÃO DO ESTADO NUTRICIONAL PRECEDENTE AO USO DE NUTRIÇÃO ENTERAL
}

\author{
Vânia Aparecida LEANDRO-MERHI', Juliana Luisi MORETE ${ }^{2}$ e \\ Maria Rita Marques de OLIVEIRA ${ }^{3}$
}

\begin{abstract}
RESUMO - Contexto - O adequado diagnóstico do estado nutricional é de vital importância para a prescrição da terapia nutricional enteral no ambiente hospitalar. Objetivo - Avaliar indicadores do estado nutricional em pacientes ingressantes na terapia nutricional enteral em uma unidade hospitalar. Métodos - Estudo transversal com 100 pacientes adultos, sendo analisado o estado nutricional de ingresso à terapia nutricional enteral, por meio do índice de massa corporal obtido do peso e estatura estimados a partir de fórmulas de predição, e de indicadores laboratoriais do estado metabólico e nutricional. Resultados - Do total, 29\% dos pacientes foram classificados como desnutridos pelo índice de massa corporal, enquanto $80 \%$ dos mesmos apresentaram albumina abaixo do valor de referência ( $<3,2 \mathrm{~g} / \mathrm{dL}$ ). Não houve diferença na distribuição das causas de base da internação entre os grupos classificados quanto ao estado nutricional pelo índice de massa corporal, prevalecendo as doenças cardiovasculares e pulmonares entre as principais causas. As concentrações abaixo dos valores de referência de albumina não foram diferentes entre os grupos classificados pelo índice de massa corporal e pelo diagnóstico de internação. Conclusão - O índice de massa corporal estimado foi indicador específico do estado nutricional, porém pouco sensível, enquanto a albumina mostrou-se mais sensível, o que reafirma a necessidade da combinação de vários indicadores para obtenção de um adequado diagnóstico nutricional.
\end{abstract}

DESCRITORES - Estado nutricional. Desnutrição. Nutrição enteral. Pacientes internados.

\section{INTRODUÇÃO}

O estudo dos indicadores da desnutrição no âmbito hospitalar é de grande relevância, já que vários trabalhos nos últimos 25 anos têm mostrado as consequências da desnutrição para os pacientes hospitalizados ${ }^{(14,26)}$. Após a internação hospitalar, cerca de $70 \%$ dos pacientes inicialmente desnutridos, sofrem uma piora gradual do seu estado nutricional ${ }^{(16)}$. Este número contribui para o aumento da morbidade e mortalidade, em até $65 \%$ dos pacientes ${ }^{(11,16)}$. Este déficit nutricional acarreta o aumento da incidência de infecções hospitalares, cicatrização de feridas mais lentas, aumentando ainda mais o tempo de internação, além de contribuir para o aumento dos custos hospitalares ${ }^{(11)}$.

A terapia nutricional enteral (TNE) tem sido empregada rotineiramente como alternativa bem sucedida para melhorar as condições nutricionais nos pacientes hospitalizados ${ }^{(24,28)}$. A manutenção e até mesmo a recuperação do estado nutricional vêm sendo encontradas com frequência entre pacientes que recebem $\mathrm{TNE}^{(14,27)}$. Entretanto, a indicação da TNE deve seguir critérios bem definidos ${ }^{(28)}$.
Entre os critérios está o aporte insuficiente de nutrientes, seja qual for o motivo. $\mathrm{O}$ aporte insuficiente de nutrientes torna-se ainda mais crítico em presença de desnutrição ${ }^{(28)}$. Daí a importância do diagnóstico nutricional, que no caso do enfermo representa um desafio, pois a desnutrição aguda nem sempre se expressa pelos indicadores antropométricos ${ }^{(15)}$. De outro lado, os indicadores bioquímicos do estado nutricional podem sofrer interferência do processo da doença, o que pode mascarar os resultados ${ }^{(15)}$. Há que se considerar ainda, as dificuldades de obtenção de medidas antropométricas de pacientes acamados e as alterações da composição corporal em água, inerentes ao processo da doença.

Para minimizar essas dificuldades, vários instrumentos de avaliação têm sido desenvolvidos e utilizados na prática clínica $^{(7,17,18)}$. Esses instrumentos têm lançado mão de aspectos subjetivos de avaliação, muitas vezes considerando não o estado de desnutrição em si, mas sim os fatores de risco para que ele se instale, como por exemplo, a dificuldade de acesso ao alimento. Dessa forma, os avanços da nutrição clínica têm nos mostrado que a avaliação do estado nutricional se torna cada

${ }^{1}$ Faculdade de Nutrição, Pontifícia Universidade de Campinas, Campinas, SP; ${ }^{2}$ Curso de Nutrição, Unversidade Metodista de Piracicaba, Piracicaba, SP; 3 Instituto de Biociências, Universidade Estadual Paulista, Botucatu, SP.

Correspondência: Vânia Ap. Leandro-Merhi - Av. Carlos Grimaldi, 1171, Quadra D, Lote 13 - Bairro: Jardim Madalena - Residencial Vila Verde - 13091-906- Campinas SP. E-mail: valm@dglnet.com.br 
vez mais imprescindível e complexa, demandando sempre novos estudos.

Considerando a importância da triagem para o adequado cuidado nutricional, o presente trabalho teve por objetivo avaliar indicadores do estado nutricional em pacientes ingressantes na TNE em uma unidade hospitalar.

\section{MÉTODOS}

Foi realizado estudo transversal com 100 pacientes adultos $(\mathrm{n}=100)$ internados com indicação de TNE em uma unidade hospitalar, entre os meses de agosto a dezembro de 2006. Foram incluídos na pesquisa todos os pacientes que estivessem recebendo TNE exclusiva, tivessem mais que 18 anos e não mais que 3 dias de internação. A coleta de informações para fins da pesquisa, só foi iniciada depois da assinatura do termo de consentimento livre e esclarecido por parte do paciente ou responsável, tendo obtido parecer favorável do Comitê de Ética em Pesquisa da Universidade Metodista de Piracicaba, SP (processo n ${ }^{\circ} 83 / 05$ ), como parte do projeto Evolução dos Indicadores do Estado Nutricional e Alimentar de Grupos específicos da População de Piracicaba e região. Para a coleta dos dados, foi utilizado um protocolo previamente estabelecido, incluindo dados de identificação pessoal, diagnóstico, indicadores antropométricos (estatura estimada, peso estimado e desejado, índice de massa corporal (IMC), circunferência do braço $(\mathrm{CB})$, prega cutânea do tríceps (PCT), circunferência muscular do braço (CMB), indicadores laboratoriais do estado metabólico e nutricional (glicemia, ureia, creatinina, hemoglobina, hematócrito e albumina).

Considerando que quase a totalidade da amostra era representada por pacientes acamados e impossibilitados de ficarem em pé para aferição de peso e estatura, essas medidas foram estimadas a partir de fórmulas de predição. Para a estimativa da estatura foi utilizada a medida da altura do joelho na fórmula de CHUMLEA et al. ${ }^{(5)}$. A estimativa de peso foi calculada a partir da CB, panturrilha e prega cutânea subescapular, utilizando-se a fórmula de CHUMLEA et al. ${ }^{(5)}$. O IMC foi obtido a partir da relação peso/estatura ${ }^{2}$ e classificado de acordo com os critérios da Organização Mundial da Saúde(28).

A CB foi aferida com o auxílio de uma fita métrica plástica graduada em centímetros, com precisão para milímetros, a PCT foi obtida a partir do paquímetro da marca Lange Skinfold Caliper ${ }^{\circledR}$ e a CMB foi calculada utilizando-se a seguinte fórmula: $\mathrm{CMB}=\mathrm{CB}-(\mathrm{PCT} x$ 0,314). Para os valores de referência de PCT foi adotado o critério estabelecido por FRISANCHO ${ }^{(12)}$. Quanto aos dados laboratoriais de glicemia, ureia, creatinina, hemoglobina e hematócrito utilizaram-se como ponto de corte os valores esperados preconizados por FLEURY MEDICINA e SAÚDE ${ }^{(10)}$. Para efeito de comparação, as variáveis quantitativas foram dicotomizadas de acordo com os pontos de corte estabelecidos. O ponto de corte adotado para a albumina foi de $3,2 \mathrm{~g} / \mathrm{dL}^{(15)}$, para a PCT foi de $16 \mathrm{~mm}$ para mulheres e $12 \mathrm{~mm}$ para os homens ${ }^{(12)}$, o ponto de corte para hematócrito foi de $40 \%$ para os homens e $35 \%$ para as mulheres, e para a hemoglobina foi de 13,5 e $12 \mathrm{~g} / \mathrm{dL}$, respectivamente ${ }^{(10)}$.

Os dados foram processados pelo programa Excel, sendo expressos por meio de estatística descritiva. As diferenças entre as médias de variáveis numéricas foram testadas pelo teste $t$ de Student, adotando-se o nível de significância de 5\% $(P<0,05)$. As diferenças entre as distribuições percentuais das variáveis nominais foram analisadas pelo teste qui ao quadrado, agrupando-se os valores quando necessário. Assim, para efeito de comparação, a frequência percentual dos indivíduos com valores de exames bioquímicos críticos ou não, foi determinada pelas categorias do estado nutricional. Em seguida, foram construídas tabelas de contigência ${ }^{(20)}$, cruzando as frequências percentuais das principais variáveis ilustrativas com as variáveis ativas, e utilizado o teste de qui ao quadrado de Pearson para avaliar o grau de independência entre as variáveis:

$$
\chi_{\mathrm{obs}}^{2}=\sum_{\mathrm{ij}} \frac{\left(\mathrm{y}_{\mathrm{ij}}-\mathrm{e}_{\mathrm{ij}}\right)^{2}}{\mathrm{e}_{\mathrm{ij}}}
$$

onde $e_{i j}=$ frequência esperada de ocorrências do cruzamento das variáveis na amostra, se estas fossem independentes. Essa estatística tem distribuição de $\chi^{2}$ com (I-1) (J-1) graus de liberdade ( $\mathrm{I}=$ número de níveis da variável $\mathrm{X}$ e $\mathrm{J}=$ número de níveis da variável Z), ou seja, o valor de $\chi^{2}$ observado foi comparado com valor tabelado a nível $P$ de erro e os graus de liberdade associados às duas categorizações. Assim, se $\chi_{\mathrm{obs}}^{2}>\chi_{\mathrm{tab}(5 \%)}^{2}(P £ 0,05)$, rejeitou-se a hipótese de independência (ou de relação) entre as variáveis a nível de $5 \%$.

\section{RESULTADOS}

As Tabelas 1 e 2 apresentam o perfil geral da amostra estudada, dividida conforme o sexo. $\mathrm{O}$ grupo foi composto por $50 \%$ dos pacientes do sexo masculino e $50 \%$ do feminino, com situações clínicas como doença do sistema nervoso central, doenças cardiovasculares, doenças pulmonares, desnutrição, nefropatias, neoplasias, sepse, traumatismo crânio-encefálico e outras. Observou-se maior prevalência das doenças cardiovasculares $(31 \%)$, seguidas pelas pulmonares $(21 \%)$

TABELA 1. Distribuição do diagnóstico entre indivíduos com indicação de TNE durante a internação $(n=100)$

\begin{tabular}{lccc}
\hline & $\begin{array}{c}\text { Sexo } \\
\text { masculino } \\
\mathbf{n}\end{array}$ & $\begin{array}{c}\text { Sexo } \\
\text { feminino } \\
\mathbf{n}\end{array}$ & $\begin{array}{c}\text { Total } \\
\mathbf{n}(\%)\end{array}$ \\
\hline Diagnóstico & 2 & & \\
$\quad$ Doença do sistema nervoso central & 15 & 16 & $31(31)$ \\
Doença cardiovascular & 11 & 10 & $21(21)$ \\
Doença pulmonar & - & 2 & $2(2)$ \\
Desnutrição & - & 2 & $2(2)$ \\
Nefropatia & 3 & - & $3(3)$ \\
Neoplasia & 1 & 3 & $4(4)$ \\
Sepse & 7 & 3 & $10(10)$ \\
Traumatismo crânio-encefálico & 11 & 14 & $25(25)$ \\
Outros & 50 & 50 & $100(100)$ \\
Total & & & \\
\hline
\end{tabular}


sem diferença estatística na distribuição proporcional entre os sexos. Nos indicadores do estado nutricional e metabólico (Tabela 2), observa-se que as mulheres apresentam IMC e PCT mais elevados que os homens. A amostra como um todo apresentou valores médios de glicemia e ureia acima dos esperados. Os valores de albumina apresentaram-se abaixo do esperado (Tabela 2).

TABELA 2. Indicadores do estado nutricional entre indivíduos com indicação de TNE durante a internação $(n=100)$

\begin{tabular}{lccc}
\hline & $\begin{array}{c}\text { Sexo } \\
\text { masculino }\end{array}$ & $\begin{array}{c}\text { Sexo } \\
\text { feminino }\end{array}$ & Total \\
& $\overline{\mathbf{X}} \pm \mathrm{DP}$ & $\overline{\mathbf{X}} \pm \mathbf{D P}$ & $\overline{\mathbf{X}} \pm \mathbf{D P}$ \\
\hline Antropometria & & & \\
$\quad$ Estatura estimada $(\mathrm{cm})(*)$ & $167,68 \pm 9,75$ & $156,26 \pm 5,11$ & $161,97 \pm 9,64$ \\
Peso estimado $(\mathrm{kg})$ & $58,75 \pm 18,25$ & $56,03 \pm 17,09$ & $57,39 \pm 17,64$ \\
Peso desejado $(\mathrm{kg})(*)$ & $65,70 \pm 10,13$ & $58,09 \pm 4,67$ & $61,90 \pm 8,73$ \\
IMC $\left(\mathrm{m}^{2} / \mathrm{kg}\right)(*)$ & $20,40 \pm 5,43$ & $22,86 \pm 6,40$ & $21,63 \pm 6,04$ \\
Circunferência do braço $(\mathrm{cm})$ & $26,44 \pm 5,11$ & $28,14 \pm 6,15$ & $27,29 \pm 5,69$ \\
Circunferência muscular do & $21,61 \pm 3,65$ & $21,47 \pm 3,82$ & $21,54 \pm 3,72$ \\
braço $(\mathrm{cm})$ & & & \\
Prega cutânea do tríceps & $14,56 \pm 7,27$ & $20,6 \pm 10,76$ & $17,58 \pm 9,63$ \\
(mm) $(*)$ & & & \\
Exames laboratoriais & & & \\
Glicemia $(\mathrm{mg} / \mathrm{dL})$ & $147,84 \pm 65,55$ & $145,86 \pm 78,43$ & $146,85 \pm 71,92$ \\
Ureia $(\mathrm{mg} / \mathrm{dL})$ & $62,88 \pm 60,47$ & $56,70 \pm 41,17$ & $59,79 \pm 51,56$ \\
Creatinina $(\mathrm{mg} / \mathrm{dL})$ & $2,16 \pm 4,89$ & $1,03 \pm 0,68$ & $1,60 \pm 3,52$ \\
Hemoglobina $(\mathrm{g} / \mathrm{dL})(*)$ & $11,56 \pm 1,99$ & $10,49 \pm 2,05$ & $11,03 \pm 2,08$ \\
Hematócrito $(\%)$ & $33,46 \pm 5,75$ & $31,38 \pm 7,47$ & $32,42 \pm 6,71$ \\
Albumina $(\mathrm{g} / \mathrm{dL})$ & $2,58 \pm 0,60$ & $2,56 \pm 0,68$ & $2,57 \pm 0,64$ \\
\hline (*) P<0,05, no reste $t$ de Student na comparacão das médias entre os sexos & &
\end{tabular}

As Tabelas 3, 4 e 5 são referentes aos indicadores do estado nutricional. Na Tabela 3 verifica-se que não houve diferença na distribuição do diagnóstico entre os pacientes divididos conforme o estado nutricional classificado pelo IMC. Nesta classificação $29 \%$ dos pacientes apresentaram-se desnutridos, $53 \%$ eutróficos e 18\% obesos (Tabela 3). A distribuição da albumina sérica não foi diferente entre os grupos classificados tanto pelo IMC, quanto pelo diagnóstico do registro de internação (Tabela 4). Os valores de concentração de albumina abaixo do ponto de corte de $3,2 \mathrm{~g} / \mathrm{dL}$ foram identificados em

TABELA 3. Estado nutricional em relação ao diagnóstico

\begin{tabular}{lccc}
\hline & \multicolumn{3}{c}{ Estado nutricional } \\
& Desnutrição & Eutrofia & Obesidade \\
& $\mathbf{n}(\%)$ & $\mathbf{n}(\%)$ & $\mathbf{n}(\%)$ \\
\hline Diagnóstico (\%) & & & \\
$\quad$ Doença do sistema nervoso central & $2(6,9)$ & - & - \\
Doença cardiovascular & $7(24,1)$ & $19(35,8)$ & $5(27,8)$ \\
Doença pulmonar & $4(13,8)$ & $10(18,8)$ & $7(38,9)$ \\
Desnutrição & $2(6,9)$ & - & - \\
Nefropatia & - & $1(1,9)$ & $1(5,6)$ \\
Neoplasia & $1(3,4)$ & $2(3,7)$ & - \\
Sepse & - & $2(3,7)$ & $2(11,1)$ \\
Traumatismo crânio-encefálico & $2(6,9)$ & $8(15,1)$ & - \\
Outros & $11(37,9)$ & $11(20,8)$ & $3(16,7)$ \\
Total & $29(100)$ & $53(100)$ & $18(100)$ \\
\hline$\chi^{2}=11,1 ;$ NS; com 5 graus de liberdade na comparação proporção entre os grupos distribuídos conforme o estado nutricional
\end{tabular}

TABELA 4. Albumina plasmática e sua correlação com o estado nutricional, diagnóstico e prega cutânea do tríceps

\begin{tabular}{|c|c|c|c|}
\hline & \multicolumn{3}{|c|}{ Albumina $(\mathrm{g} / \mathrm{dL})$} \\
\hline & $>3,2$ & $<3,2$ & Total \\
\hline \multicolumn{4}{|l|}{ Estado nutricional (\%) } \\
\hline Desnutridos & 5 & 23 & \multirow{4}{*}{$\begin{array}{c}\qquad \chi^{2}=1,25 \\
\text { NS } \\
2 \text { graus de liberdade }\end{array}$} \\
\hline Eutróficos & 13 & 42 & \\
\hline Obesos & 2 & 15 & \\
\hline Total & 20 & 80 & \\
\hline \multicolumn{4}{|l|}{ Diagnóstico (\%) } \\
\hline Doença do sistema nervoso central & - & 2 & \multirow{10}{*}{$\begin{array}{c}\qquad \chi^{2}=9,7 \\
\text { NS } \\
7 \text { graus de liberdade }\end{array}$} \\
\hline Doença cardiovascular & 8 & 23 & \\
\hline Doença pulmonar & 5 & 16 & \\
\hline Desnutrição & 1 & 1 & \\
\hline Nefropatia & - & 2 & \\
\hline Neoplasia & 1 & 2 & \\
\hline Sepse & 1 & 3 & \\
\hline Traumatismo crânio-encefálico & 1 & 9 & \\
\hline Outros & 3 & 22 & \\
\hline Total & 20 & 80 & \\
\hline \multicolumn{4}{|l|}{ Prega cutânea do triceps (mm) } \\
\hline Sexo feminino & & & \multirow{3}{*}{$\begin{array}{c}\chi^{2}=0,28 \\
\text { NS } \\
1 \text { grau de liberdade }\end{array}$} \\
\hline$>16$ & 7 & 15 & \\
\hline$<16$ & 7 & 21 & \\
\hline Sexo masculino & & & \multirow{4}{*}{$\begin{array}{c}\chi^{2}=3,83 \\
P=0,050 \\
1 \text { grau de liberdade }\end{array}$} \\
\hline$>12$ & 1 & 21 & \\
\hline$<12$ & 7 & 21 & \\
\hline Total & 22 & 78 & \\
\hline
\end{tabular}

$80 \%$ dos pacientes. Apenas $22 \%$ dos pacientes apresentaram PCT em valores abaixo do ponto de corte adotado. A Tabela 5 mostra a distribuição do hematócrito e hemoglobina entre os pacientes divididos por sexo, estado nutricional classificado pelo IMC e pelo diagnóstico. Houve diferença na distribuição dos valores hematológicos e estado nutricional em ambos os sexos, com maior prevalência de adequação entre os pacientes com menor peso (Tabela 5).

\section{DISCUSSÃo}

A TNE tem sido considerada sempre que possível como o método de escolha para pacientes internados, principalmente os de Unidade de Terapia Intensiva (UTI), pois estes não possuem condições de alimentação por via oral suficientes para atingir o mínimo de suas necessidades energéticas, normalmente aumentadas pelo estresse fisiológico ${ }^{(28)}$. A TNE precoce beneficia o estado nutricional, reduz o tempo de internação hospitalar e proporciona melhor reembolso diário ao Sistema de Saúde hospitalar ${ }^{(27)}$. No presente estudo, a introdução da TNE ocorreu num intervalo de até 72 horas da internação e foi indicada para um grupo de pacientes com elevada proporção de pacientes desnutridos, $29 \%$ na classificação pelo IMC estimado e $80 \%$ na classificação pela albumina. Lembrando que a albumina sérica é indicador do 
TABELA 5. Indicadores laboratoriais (hematócrito/hemoglobina) e sua relação com o estado nutricional e diagnóstico

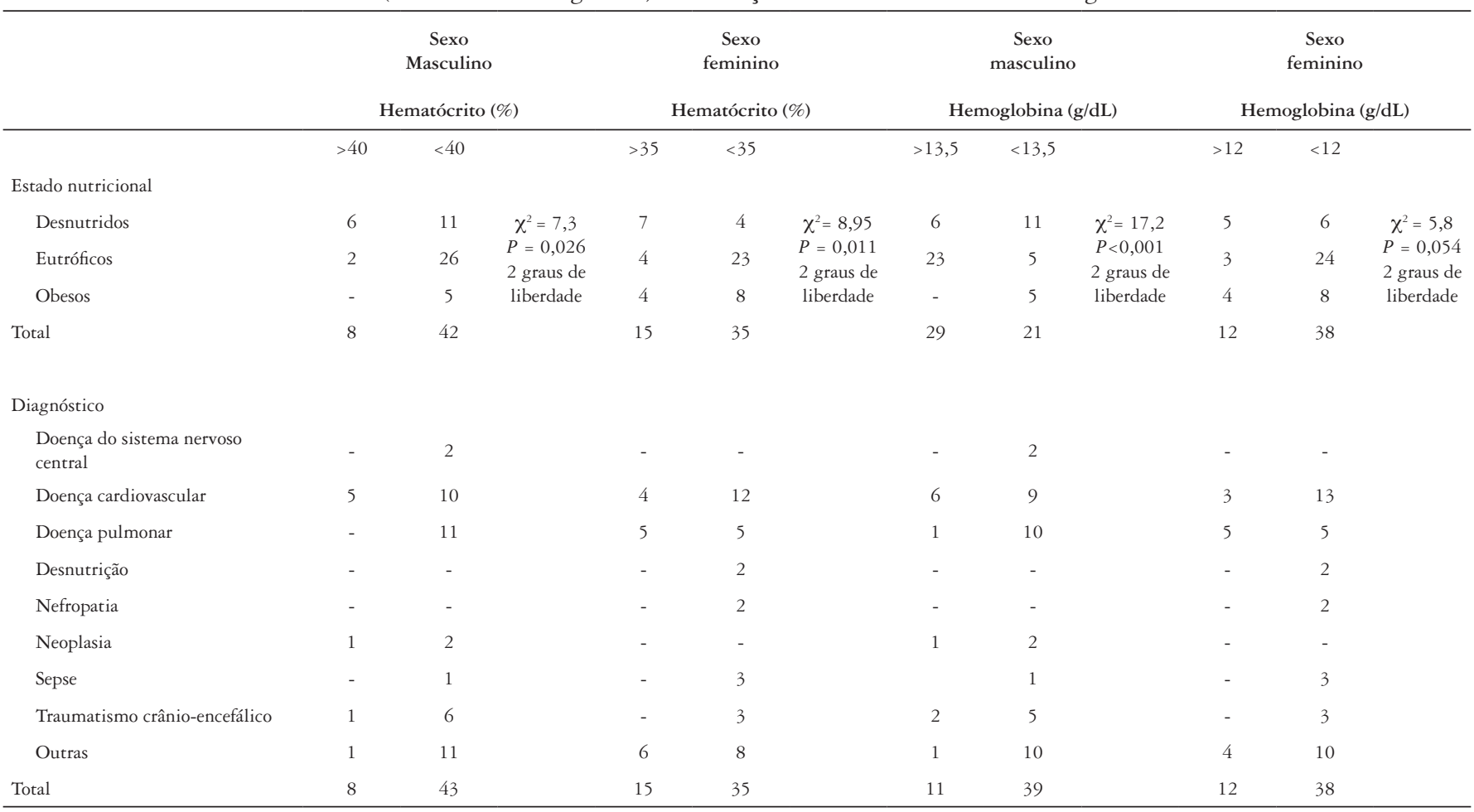

estado nutricional, cujos déficits se manifestam comumente no estresse catabólico, associado ao consumo deficiente de proteínas e energia $^{(15)}$. No presente estudo foram verificados valores médios elevados de glicose, ureia e creatinina, o que pode caracterizar o grupo como população em intenso estado catabólico. Com relação às situações clínicas mais frequentes, observaram-se as doenças cardiovasculares; outros trabalhos na mesma região do Estado de São Paulo têm encontrado as neoplasias como as doenças de maior prevalência ${ }^{(21)}$.

A equipe profissional de cuidado ao paciente tem importante papel na indicação e administração adequada da $\mathrm{TNE}^{(24)}$. O conhecimento do estado nutricional do paciente no momento de se iniciar a TNE possibilita a sua adequada prescrição ${ }^{(9)}$, assim como as avaliações periódicas permitem as adequações da prescrição conforme a evolução do estado nutricional do paciente que está recebendo a TNE. Nesse sentido, as proteínas viscerais, dependendo de sua meia vida, são indicadores do estado nutricional mais ou menos úteis para avaliar o estado nutricional na triagem para a $\mathrm{TNE}$ ou no resultado da $\mathrm{TNE}^{(1,15)}$. As proteínas viscerais de curta meio vida, como por exemplo a pré-albumina, são importantes marcadores da evolução do estado nutricional durante o período de TNE; já a albumina, com meia vida de 20 dias, constitui-se num importante indicador do estado nutricional para o momento da triagem do paciente para o cuidado nutricional ou para avaliação em períodos mais $\operatorname{longos}^{(15)}$. O interesse do presente trabalho foi conhecer o estado nutricional no ingresso à TNE.
A prevalência de desnutrição no ambiente hospitalar ${ }^{(13)}$ tem variado em função do tipo de indicador nutricional utilizado, o tipo de hospital, a população estudada e o critério adotado. Em estudo realizado por WAITZBERG et al. ${ }^{(25)} \mathrm{em}$ hospitais brasileiros, no qual foi utilizada a Avaliação Global Subjetiva (AGS), foram encontrados 31,8\% dos pacientes com desnutrição. Em uma população proveniente da mesma região geográfica daquela do presente estudo, GARCIA et al. ${ }^{(14)}$ encontraram $23,1 \%$ de desnutrição entre os pacientes internados, classificados pelo IMC. Esses valores foram inferiores aos resultados do presente estudo, em que $29 \%$ dos pacientes encontraram-se desnutridos ao serem classificados pelo IMC. Entretanto, todos os pacientes desta série estavam recebendo TNE, podendo pressupor-se que sejam pacientes mais debilitados que a população geral enferma. Neste estudo, o IMC mostrou-se um indicador muito específico e pouco sensível à desnutrição pois, se comparadas às proporções, a albumina em valores abaixo de 3,2 g/dL, classifica $80 \%$ dos pacientes com desnutrição.

A albumina é um importante marcador para identificação do estado nutricional, principalmente quando utilizada conjuntamente a outros indicadores. LOPEZ et al. ${ }^{(19)}$ verificaram que em 250 pacientes selecionados, 54,8\% apresentaram desnutrição protéica; quando analisados somente pela antropometria, apenas 2,8\% apresentaram-se desnutridos. Foi verificado, em estudo com cirurgia pancreática na idade avançada, que a perda de peso associada às baixas concentrações séricas de albumina são fatores de risco independentes para 
complicações pós-operatórias ${ }^{(3)}$. É importante salientar que a perda de peso não é identificada com aferição pontual do IMC e que um indivíduo que tenha perdido peso não intencionalmente, pode apresentar ainda IMC dentro ou acima dos valores esperados ${ }^{(7)}$. Nesse caso, a albumina pode contribuir como importante indicador do estado nutricional.

Contudo, a diminuição dos valores de albumina sérica em pacientes hospitalizados por longo período pode melhor refletir condições metabólicas ou inflamatórias do que propriamente o estado nutricional. A variabilidade da hipoalbuminemia pode ser influenciada pela desnutrição, pelo estado de hidratação do paciente, redução da síntese, estresse orgânico, inflamação, entre outras ${ }^{(15)}$. A principal dificuldade de se utilizar a albumina como marcador nutricional isolado é sua relação direta com a inflamação. Pacientes bem nutridos podem apresentar baixos níveis séricos de albumina em situações de estresse orgânico, como a inflamação ${ }^{(8)}$. Porém, existe uma interdependência entre os parâmetros bioquímicos e nutricionais refletidos pela albumina ${ }^{(8)}$. BRUGLER et al. ${ }^{(4)}$ comprovam a necessidade da junção de indicadores nutricionais, como modelo diagnóstico para a utilização em pacientes de risco nutricional, apontando que as características que melhor se correlacionaram com as complicações relacionadas à desnutrição, foram o baixo consumo energético, as baixas concentrações de albumina e hemoglobina e a baixa contagem de linfócitos.

Constatou-se ainda que grande parcela da população estudada ( $42 \%$ do sexo masculino e $35 \%$ do sexo feminino) apresentou baixos valores plasmáticos de hematócrito, bem como de hemoglobina. Os baixos valores hematológicos podem indicar deficiência por perda ou menor síntese, mas também podem ser indicadores de diluição por questões relativas ao desequilíbrio hemodinâmico ${ }^{(15)}$. No presente estudo, a maior proporção de homens e mulheres com hematócrito acima do ponto de corte eram de pacientes com baixo peso, que pode ser indício de desidratação, o que se torna mais um fator a depor contra o uso isolado do IMC para identificar o estado nutricional do paciente hospitalizado.

Entretanto, o conhecimento do peso e da estatura de pacientes é de grande importância para a prática clínica, não só para a avaliação do estado nutricional, mas também para a prescrição de medicamentos e estimativa da necessidade nutricional. Mas, a obtenção destas medidas em pacientes acamados nem sempre é possível nos serviços de saúde ${ }^{(23)}$. Desde a década de 80 as fórmulas de predição de peso e estatura vêm sendo utilizadas como alternativas para idosos e pacientes acamados ${ }^{(5)}$. As fórmulas propostas por CHUMLEA et al. ${ }^{(5)}$ para a população americana são as mais conhecidas e utilizadas. No entanto, tem se recomendado cuidado no uso indiscriminado dessas fórmulas, pois as características físicas das populações diferem de uma para outra ${ }^{(15)}$. No Brasil as fórmulas de CHUMLEA et al. ${ }^{(5)}$ apresentaram concordância com o peso aferido em Fortaleza ${ }^{(23)}$, mas não em Ribeirão Preto (22). Neste último, o peso foi superestimado pelas fórmulas. É possível que também no presente estudo o peso tenha sido superestimado, embora o IMC médio tenha sido mais próximo do estudo de Fortaleza, em torno de $22 \mathrm{~kg} / \mathrm{m}^{2}$.

Dada as limitações dos métodos antropométricos e bioquímicos, reforça-se a importância da combinação de vários indicadores para a identificação de pacientes de risco $^{(2)}$. Além disto, a triagem do enfermo acamado para o cuidado nutricional tem se beneficiado com as abordagens que levam em conta critérios subjetivos de avaliação do risco nutricional.

Num estudo multicêntrico ${ }^{(6)}$, foi avaliada a prevalência de desnutrição em pacientes hospitalizados e a relação de diferentes indicadores nutricionais com o tempo de internação e mortalidade, sendo encontrado que, pela Mini Avaliação Nutricional (MAN), 23,9\% dos pacientes estavam desnutridos e $50 \%$ apresentaram risco de desnutrição. A análise de correlação com o tempo de internação apontou associação positiva com a MAN, concluindo alta prevalência de desnutrição entre pacientes hospitalizados e declínio da mortalidade conforme o aumento do escore da MAN. Anterior a MAN, a Avaliação Global Subjetiva $^{(7)}$ vem sendo amplamente utilizada na prática clínica e constitui-se uma abordagem que leva em conta a perda recente de peso, a quantidade e qualidade do consumo alimentar, as condições do trato digestório, as demandas metabólicas e as condições físicas e funcionais. Essa avaliação, por ser subjetiva, depende de experiente "olho clínico" e daí a necessidade de que seja realizada por profissionais treinados a partir de protocolos bem estabelecidos. Já o "Nutritional Risk Screening" (NRS, 2002), proposto por KONDRUP et al. ${ }^{(18)}$, foi baseado no conceito de que a terapia nutricional deve ser indicada para pacientes com a ingestão diminuída de nutrientes e perda ponderal associada às demandas energéticas aumentadas pela condição clínica. Trata-se de instrumento mais específico à indicação da TNE e de mais fácil aplicação. Nesses instrumentos de avaliação subjetiva, o IMC é levado em consideração, mas deles leva em conta as concentrações séricas de albumina, um indicador bioquímico de fácil obtenção e frequentemente associado à desnutrição em condição de estresse ${ }^{(15)}$.

Concluindo, o IMC obtido do peso e estatura estimados a partir de equações de predição, foi indicador específico do estado nutricional, porém pouco sensível, enquanto a albumina mostrou-se mais sensível e pouco específica, o que reafirma a necessidade da combinação de vários indicadores para obtenção de adequado diagnóstico nutricional. 
Leandro-Merhi VA, Morete JL, Oliveira MRM. Assessing nutritional status before introducing enteral nutrition. Arq Gastroenterol. 2009;46(3):219-24.

ABSTRACT - Context - A proper diagnosis of the nutritional status is of the utmost importance for prescribing enteral nutrition therapy in the hospital environment. Objective - To assess nutritional status indicators of patients about to receive enteral nutrition therapy in a hospital unit. Methods - This cross-sectional study examined the nutritional status of 100 adult patients before they were introduced to enteral nutrition therapy by calculating their body mass index . Their height and weight were estimated by prediction formulas and laboratory indicators of nutritional and metabolic statuses. Results - Almost one-third (29\%) of the patients were classified as malnourished according to their body mass index, while $80 \%$ of them had low albumin values $(<3.2 \mathrm{~g} / \mathrm{dL})$. When patients were grouped according to body mass index, the distribution of the reasons for hospitalization did not differ between the groups. Cardiovascular and pulmonary diseases prevailed as the main reasons for hospitalization. When patients were grouped according to body mass index and diagnosis upon admission, the rates of low albumin concentration, i.e., concentration below the reference value, did not differ between the groups. Conclusion - The estimated body mass index was a specific indicator of nutritional status but lacked sensitivity. Meanwhile, albumin was more sensitive, demonstrating that it is necessary to use many indicators in combination to diagnose nutritional status properly.

HEADINGS - Nutritional status. Malnutrition. Enteral nutrition. Inpatients.

\section{REFERÊNCIAS}

1. Almeida JC, Kuhmmer R, Sponchiado E, Laflor CM, Weber B. Desenvolvimento de um instrumento de triagem nutricional para avaliação do risco de desnutrição na admissão hospitalar. Rev Bras Nutr Clin. 2008;23:28-33.

2. Blanco, LV, Rausell GL, Vidal VJ, Pérez-Crespo CG, Navalón CI, Sirvent MCM, Panella MM, Iñigo SR. Valoración nutricional al ingreso hospitalario: iniciación al estúdio entre distintas metodologías. Nutr Hosp. 2006;21:163-72.

3. Bozzetti F, Gianotti L, Braga M, Carlo VD, Mariani L. Postoperative complications in gastrointestinal cancer patients: the joint role of the nutritional status and the nutritional support. Clin Nutr. 2007;26:698-709.

4. Brugler L, Stankovic AK, Schlefer M, Bernstein L. A simplified nutrition screen for hospitalized patients using readily available laboratory and patient information. Nutrition. 2005;21:650-8.

5. Chumlea WC, Guo S, Roche AF, Steinbaugh ML. Prediction of body weight for non ambulatory elderly from anthropometry. J Am Diet Assoc. 1988;88: 564-8.

6. De Luis D, Lopez Guzman A. Nutritional status of adult patients admitted to internal medicine departments in public hospitals in Castilla y Leon, Spain. A multi-center study. Eur J Intern Med. 2006;17:556-60.

7. Detsky AS, McLaughlin JR, Baker JP, Johnston N, Whittaker S, Mendelson RA, Jeejeebhoy KN. What is subjective global assessmente of nutritional status? JPEN J Parenter Enteral Nutr. 1987;11:8-13.

8. Don BR, Kaysent G. Poor nutritional status and inflammation. Serum albumin: relationship to inflammation and nutrition. Sem Dialysis. 2004;17:432-7.

9. Fernández CC, González IG, Juárez FMA, Figueiras PG, Espiñeira RT, Cuesta BS, Huete AA, Deibe RM. Detección de malnutrición al ingreso en el hospital. Nutr Hosp. 2003;18:69-74.

10. Fleury Medicina e Saúde. Manual de Exames 2008/2009. São Paulo: Laboratório Fleury; 2008.

11. Fontoura CSM, Oliveira DC, Londero LG, Vieira RM. Avaliação nutricional do paciente crítico. Rev Bras Terap Intens. 2006;18:298-306.

12. Frisancho AR. Anthropometric standards for the assessment of growth and nutritional status. Ann Arbor: The University of Michigan Press; 1990. 189p.

13. Fuchs V, Mostkoff D, Gutiérrez Salmeán G, Amancio O. Estado nutricio en pacientes internados en un hospital público de la ciudad de México. Nutr Hosp. 2008;23:294-303.

14. Garcia RWD, Leandro Merhi VA, Pereira AM. Estado nutricional em pacientes internados em clínica médica. Rev Bras Nutr Clín. 2004;19:59-63.
15. Gibson RS. Principles of nutritional assessment. 2nd ed. New York: Oxford University Press; 2005. 908p.

16. Goiburu ME, Goiburu MM, Bianco H, Díaz JR, Alderete F, Palacios MC, Cabral V, Escobar D, López R, Waitzberg DL. The impact of malnutrition on morbidity, mortality and length of hospital stay in trauma patients. Nutr Hosp. 2006;21:604-10.

17. Guigoz Y, Garry JP. Mini nutritional assessment: a practical assessment tool for grading the nutritional state of elderly patients. Facts Res Gerontol. 1994;Suppl.2:15-59.

18. Kondrup J, Rasmussen HH, Hamberg O, Stanga Z, Ad Hoc ASPEN Working Group. Nutritional risk screening (NRS 2002): a new method based on a analysis of controlled clínical trials. Clin Nutr. 2003;22:321-36.

19. López MAS, Herrera RMT, Cruz AJ, Espinosa RO, Medina T, Martínz CL. Prevalencia de desnutrición em pacientes ingresados em un hospital de rehabilitación y traumatologia. Nutr Hosp. 2005;20:121-30.

20. Pimentel-Gomes F. Curso de estatística experimental. $14^{\mathrm{a}}$ ed. São Paulo: Livraria Nobel; 2000.

21. Prieto DB, Leandro-Merhi VA, Mônaco DV, Lazarini ALG. Intervenção nutricional de rotina em pacientes de um hospital privado. Rev Bras Nutr Clín. 2006;21:181-7.

22. Rabito EI, Vannucchi GB, Suen VMM, Castilho Neto LL, Marchini JS. Weight and height prediction of immobilized patients. Rev Nutr. 2006;19:655-61.

23. Sampaio HAC, Melo MLP, Almeida PC, Benevides ABP. Aplicabilidade das fórmulas de estimativa de peso e altura para idosos e adultos. Rev Bras Nutr Clin. 2002; 17:117-21.

24. Schieferdecker MEM, Silva A, Campos D. Capacidade da terapia nutricional enteral em fornecer as necessidades calórico-protéicas de pacientes hospitalizados. Rev Bras Nutr Clin. 2003;18:113-8.

25. Waitzberg DL, Caiaffa WT, Correia MITD. Hospital malnutrition: the Brazilian national survey (IBRANUTRI): a study of 4000 patients. Nutrition. 2001;17:573-80.

26. Waitzberg DL, Campos AC. Nutrition support in Brazil: past, present, and future perspectives. JPEN J Parenter Enteral Nutr. 2004;28:184-91.

27. Watanabe S, Cukier C, Magnoni D, Guimaräes RN, Urenhiuki KL, Rauba A. Nutrição enteral precoce reduz o tempo de internação hospitalar e melhora o reembolso diário do Sistema Único de Saúde (SUS) ao hospital. Rev Bras Nutr Clin. 2002;17:47-50.

28. World Health Organization. Physical status: the use and interpretation of anthropometry. Geneve; 1995. 452p.

Recebido em 6/10/2008. Aprovado em 15/1/2009. 